\title{
Arthrokinematische Adjustierung des Beckenrings
}

\author{
Dorin Ritzmann, Fachärztin Gynäkologie und Geburtshilfe, Dietikon, Schweiz
}

\section{$20 \%$ der Schwangeren sind von einer Beckenringdysfunktion betroffen und bei ca. 3-5\% chronifizieren die Beschwerden. Mit der arthrokinematischen Adjustierung des Beckenrings kann den Frauen in der Schwangerschaft, während und nach der Geburt geholfen werden.}

\begin{abstract}
Was geht schief? Federnde Böden, ein frei schwingendes Becken und eine kräftige Muskulatur sind nicht mehr überall gegeben. Asphalt und Betonböden korrelieren mit einer chronischen Überlastung der Beckengelenke, die dann bei zusätzlichen Risikofaktoren wie monotone Arbeit, jugendliches Alter, Sport, Hormonbehandlungen und hohe Parität in eine Dysfunktion übergehen kann [1, 2, 3, 4,5]. Die Beckenringdysfunktion, auch Sakro-Iliakal-Gelenk-Syndrom (SIG-Syndrom, englisch Pelvic Joint Dysfunction) genannt, ist ein „hausgemachtes“ Problem der westlichen Lebensweise. Es tritt bei $20 \%$ aller Schwangeren auf und ist bei etwa 3-5\% aller Schwangereren chronifiziert [6].
\end{abstract}

Von Bruno Maggi, Allgemeinmediziner und Manualtherapeut in Zürich, lernte ich die Diagnostik und Therapie der Beckenringdysfunktion. In der Zwischenzeit haben wir die Technik gemeinsam verfeinert und nennen unser Vorgehen arthrokinematische Adjustierung des Beckenrings. Es handelt sich um eine einfach durchzuführende, sichere und ungefährliche Methode.

Unter der Geburt ist die Adjustierung des Beckenrings von großer Bedeutung, denn die Beckenringdysfunktion ist eine häufige Ursache von

- Übertragungen,

- zunehmenden Schmerzen,

- sekundärer Wehenschwäche,
- fehlendem Tiefertreten des kindlichen Kopfes und

- Folgeoperationen.

\section{Funktionelle Anatomie, Physiologie und Pathophysiologie}

\section{Wie gehen wir frei?}

Wenn wir den menschlichen Körper als ein Musikinstrument betrachten, können wir Becken, Rumpf und Brustkorb als einen mit Flüssigkeit und Luft verwobenen Klangkörper erkennen. Schall entsteht durch eine mechanische Welle von kleinsten Druck- und Dichteschwankungen in einem elastischen Medium, wie es unser Körper ist. Durch unseren Gang entstehen mechanische Wellen. Auf federndem Boden kommt es zu fließenden, auf harten Böden zu kurzen, abrupt wechselnden Schwingungen, die die Wirbelsäule und die inneren Organe, die Lymphe, das Herz und den Kopf bewegen. Die Körperwellen können wir akustisch nicht wahrnehmen, da wir nur einen kleinen Frequenzbereich von Schallwellen hören. Die positiven Auswirkungen des federnden Gehens können jedoch nachgewiesen werden. Bekannt sind Verbesserungen von Depressionen, chronischen Schmerzen, Reizdarmsyndrom und Bluthochdruck $[8,9]$.

\section{Was ist Arthrokinematik?}

Der Begriff Arthrokinematik setzt sich aus Arthros, das Gelenk, und Kinematik, die Bewegungslehre, zusammen. Arthrokinematik bedeutet also die Lehre von der Gelenksbewegung. Sie ist ein fester Bestandteil von verschiedenen manuellen Lehren. Es werden unterschiedliche Techniken damit bezeichnet, die alle auf eine verbesserte Bewegung der Gelenksflächen zueinander abzielen. Die arthrokinematische Beckenringadjustierung zeichnet sich durch eine sehr sanfte, minimale Dehnung der Sakro-Iliakal-Gelenke aus, die damit wieder ihre physiologische Position erreichen können.

Der Beckenring ist ein knöchernes System mit 3 Gelenken, stabilisiert durch 3 ligamentäre Strukturen und gehalten von einem inneren sowie 2 äußeren Muskelgruppen [10, 11, 12] (Abb. 1). Er bewegt sich bei jedem Schritt, beim Aufrichten und Bücken, beim Drehen der Wirbelsäule im Gehen, Sitzen und Liegen $[13,14]$.

Schwingt das Sakrum frei in den SIG, ist auch die vaskuläre und neurologische Versorgung der Beckenorgane perfekt. Liegt eine länger dauernde Dysfunktion 


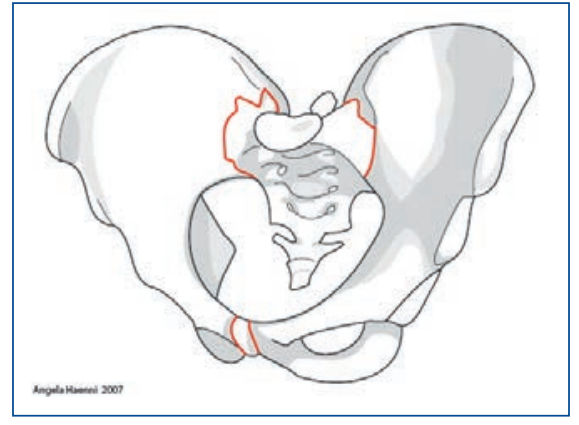

Abb. 1 Die drei Beckenringgelenke: Symphyse und zwei SIG.

vor, können Darm, Blase und Genitalorgane leiden. So können Obstipation, Reizblase und verminderte Fruchtbarkeit oft mittels einer Adjustierung des Beckenrings verbessert werden.

\section{Der knöcherne Beckenring}

Am häufigsten blockieren die SIG, weil sie beweglicher sind als die Symphyse. Da es sich um einen Ring handelt, kann sich die Spannung, meist als Schmerz wahrgenommen, auf das gegenüberliegende SIG oder die Symphyse verlagern. Typisch ist hier die Bewegungseinschränkung, die sich in einem einseitigen Hinken als Schonhaltung zeigen kann wie auch in Ausweichmanövern beim Bücken und Aufstehen [15].

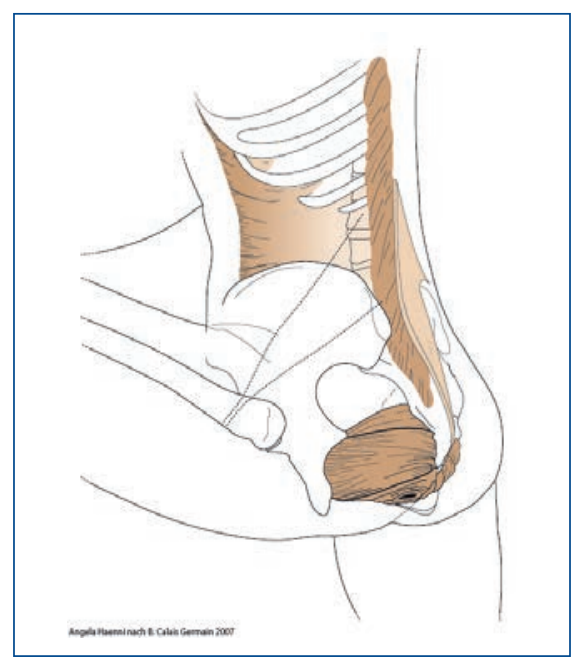

Abb. 3 Die innere Muskel- und Fasziengruppe (M. transversus abdominis, thorakodorsale Faszie, M. multifidus, M. psoas, M. levator ani, M. pubococcygeus).

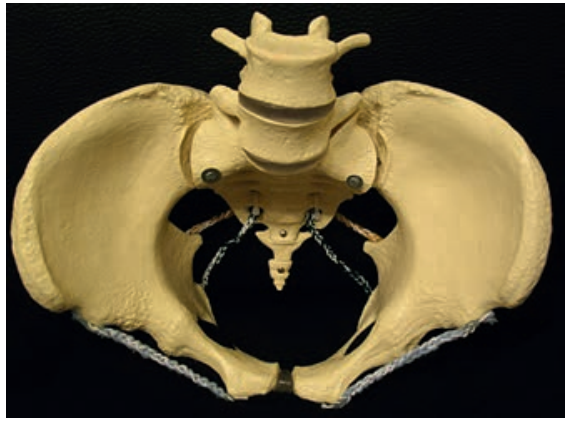

Abb. 2 Die 3 ligamentären Strukturen: Lig. inguinalia, Lig. sacrospinalia, Lig. sacrotuberalia.

Geburtsprobleme können auftreten wie zunehmende Kreuz- und Symphysenschmerzen, asymmetrisches bzw. fehlendes Tiefertreten des Kindes [16]. Eine Blockade des Beckenrings kann nach meiner Erfahrung auch an der Stimme erkannt werden: die Stimme der Gebärenden klingt gepresster, enger und höher, beim Reden, Rufen, Schreien, Summen und Singen.

\section{Die ligamentäre Stabilität}

Der knöcherne Beckenring ist neben den direkten ligamentären Gelenkverbindungen über die Sakro-Iliakal-Gelenke und die Symphyse beidseits je durch das Ligamentum inguinale, das Lig. sacrospinale und das Lig. sacrotuberale stabilisiert (Abb. 2).

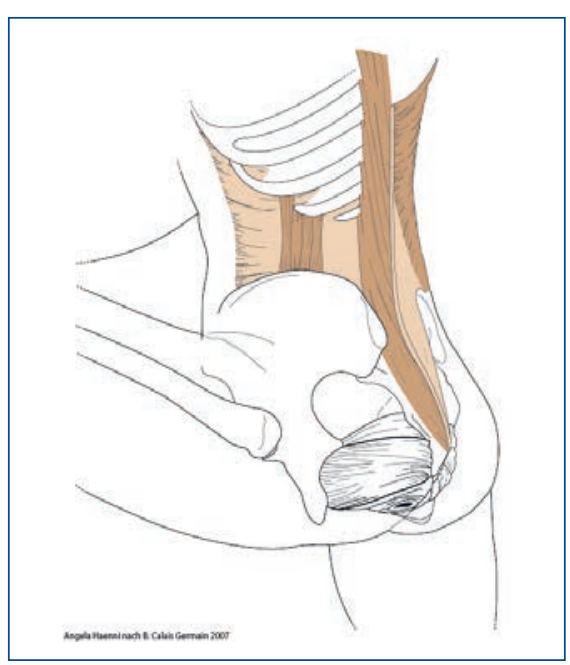

Abb. 4 Die äußere kraniale Muskelgruppe (M. quadratus lumborum, M. erector spinae, M. latissimus dorsi).
Bei einer länger dauernden Beckenringdysfunktion können eines oder mehrere dieser Bandsysteme überlastet werden und schmerzhaft anschwellen. Typisch sind hier Schmerzen im tiefen Unterbauch, Schmerzen bei der Penetration, starke Schmerzen beim Tiefertreten des kindlichen Kopfes sowie asynklitische und deflektierte Lagen während der Geburt.

Eine PDA kann sowohl die Schmerzen als auch einen hohen Muskeltonus beheben. Da dabei auch die Stabilisierung der Gelenke durch die Lockerung des Muskelmantels wie der muskulären Fasern der Ligamente vermindert wird, kann es bei Lagerungen mit passiver Bewegung des Beckenrings zu versehentlichen Blockierungen und Deblockierungen des Beckenringes kommen.

Die PDA kann durch die Lockerung der Muskulatur und Ligamente spontane Blockaden, aber auch Deblockaden des Beckenrings auslösen.

\section{Das muskuläre Schutzsystem}

Der Beckenring ist das muskuläre Zentrum des Körpers (Abb. 3-5). Lediglich die Hand-, Fuß- und Gesichtsmuskeln sind nicht direkt oder indirekt mit dem

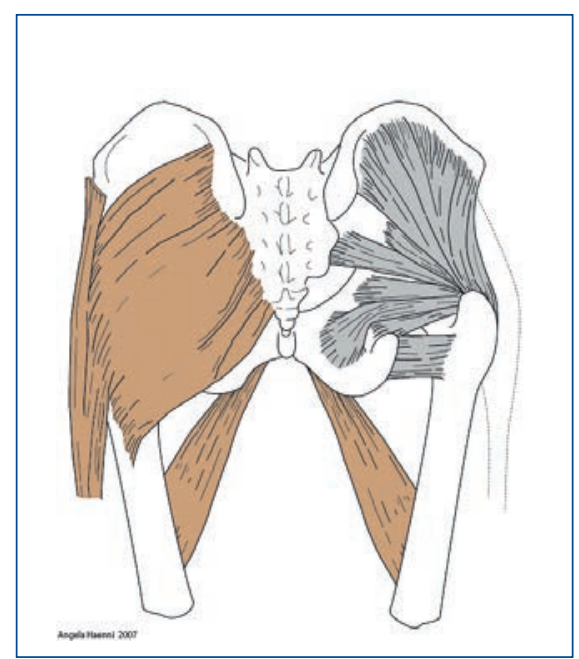

Abb. 5 Die äußere kaudale Muskel- und Fasziengruppe (Mm. glutaeus minimus, medius und maximus, M. piriformis, M. adductor femoris, Tractus iliotibialis). 
Beckenring verbunden. Ist der Beckenring blockiert, werden sich mit der Zeit eine oder mehrere der stabilisierenden Muskelgruppen anspannen, überlasten,

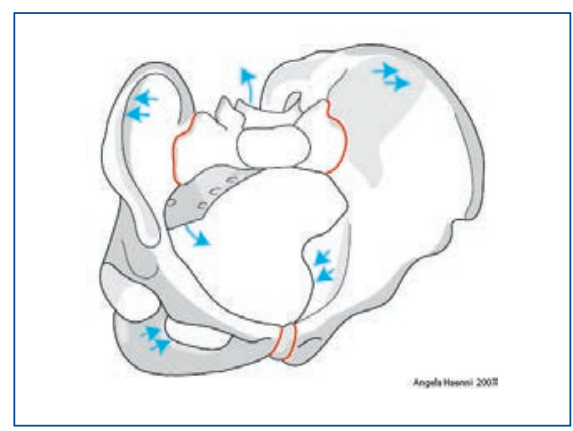

Abb. 6 Beckenringbewegung beim Aufrichten/Eröffnen.

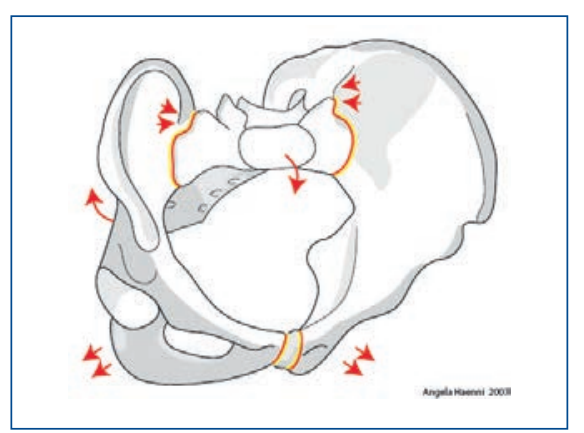

Abb. 7 Beckenringbewegung beim Bücken/Gebären.

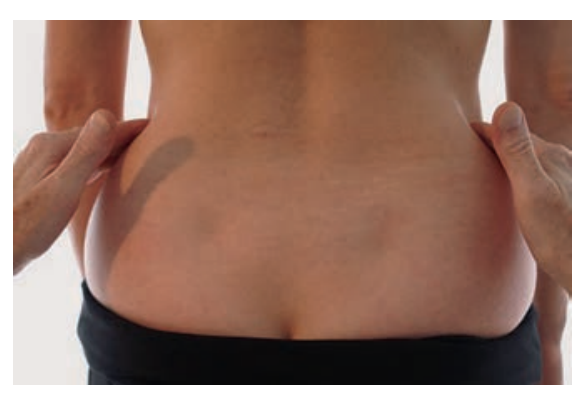

Abb. 8 Die Hände auf die Cristae iliacae legen.

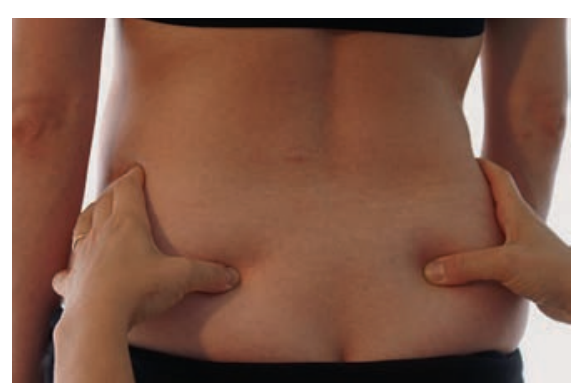

Abb. 9 Die Daumen unter die Spinae iliacae posteriores superiores schieben. entzündlich kontrahieren und ggf. sogar ihre Funktion ganz einstellen.

Typisch sind Glutäalschmerzen, die oft Tage nach einer SIG-Blockierung auftreten sowie pseudoradikuläre Ausstrahlungen entlang des $\mathrm{N}$. ischiadicus, eine Adduktorenschwäche, Schulterschmerzen, Nackenschmerzen und Drehschmerzen nachts im Schlaf, die nach einigen Wochen auftreten $[17,18]$.

Das komplexe Bewegungsmuster des Beckenrings beim Gehen, Aufrichten und Bücken bzw. beim Gebären kann mit dem Öffnen und Schließen einer Blume verglichen werden: Es ist ein dreidimensionales Geschehen. Beim Aufrichten führt das leichte dorsale Kippen des Sakrums zu einer seitlichen Öffnung der Iliakalschaufeln und einem Zusammenziehen der Sitzbeine (Abb.6). Beim Bücken führt das leicht ventrale Kippen des Sakrum umgekehrt zu einem sich Annähern der Iliakalschaufeln und einem Öffnen der Sitzbeine [19] (Abb. 7).

\section{Diagnostik der Beckenringdysfunktion}

Die Diagnostik dauert nur wenige Minuten. Leitsymptom ist die asymmetrische Beweglichkeit des Beckenrings. Zwei Tests genügen:

- das Vorlaufphänomen im Stehen und

- der Beinlängendifferenz-Test im Liegen.

Oft wende ich beide Tests an, da sie nicht exakt dasselbe messen, unter der Geburt genügt jedoch einer dieser Tests.

Diagnostik und Therapie sollten in einem praktischen Kurs erlernt und geübt werden.

\section{Das Vorlaufphänomen im Stehen}

Lassen Sie die Betroffene barfuß (oder in Socken) vor sich stehen und betrachten die Symmetrie von Schultern, Wirbelsäule und Beckenschaufeln. Legen Sie die
Hände seitlich auf die Beckenschaufeln (Cristae iliacae) und beachten deren Höhensymmetrie (Abb. 8). Bei unterschiedlicher Höhe sollten Sie an eine Beckenverwringung (Dislokation der IleumSchaufeln) oder an eine Beinlängendifferenz denken.

Nun schieben Sie die Daumen unter die Spinae iliacae posteriores superiores und bitten die Frau sich etwa 30 Grad zu bücken (Abb. 9, 10). Liegt ein asymmetrisches Verschieben der Spinae vor, ist eine Beckenringdysfunktion vorhanden.

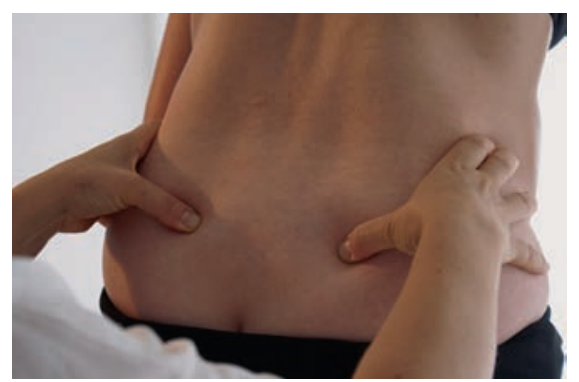

Abb. 10 Die Daumen belassen und die Betroffene bitten, sich langsam etwa 30 Grad zu bücken, dabei auf die Symmetrie der Spinae achten.

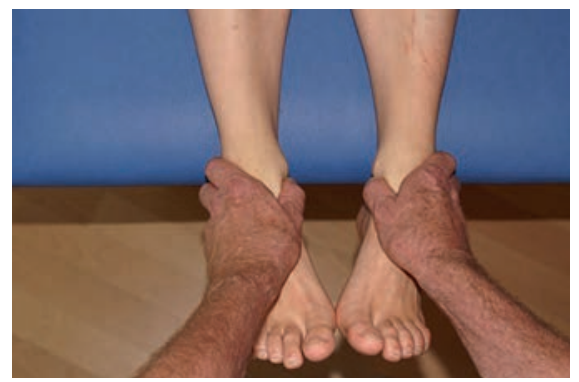

Abb. 11 Die Innenknöchel werden vor dem Aufsitzen ausgerichtet.

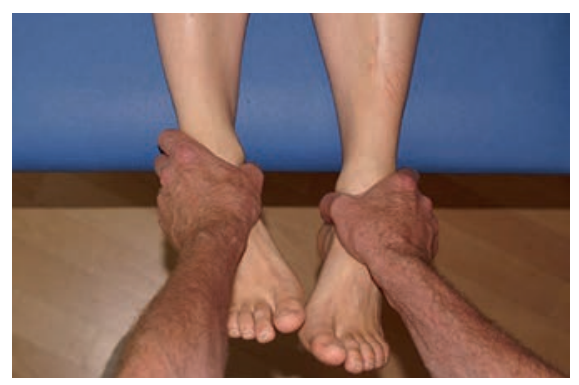

Abb. 12 Asymmetrische Knöchellage. Die Innenknöchel sind leicht verschoben: Es liegt eine Beckenringdysfunktion vor. 


\section{Der Beinlängendifferenz-Test im Liegen}

Die Betroffene legt sich auf den Rücken. Die Innenknöchel werden auf dieselbe Höhe ausgerichtet (Abb. 11). Sofort wird die Betroffene an beiden ausgestreckten Armen langsam in eine sitzende Position aufgerichtet. Behalten Sie dabei die Innenknöchel im Auge und kontrollieren Sie im Sitzen. Liegt eine Asymmetrie vor, ist eine Beckenringdysfunktion vorhanden (Abb. 12).

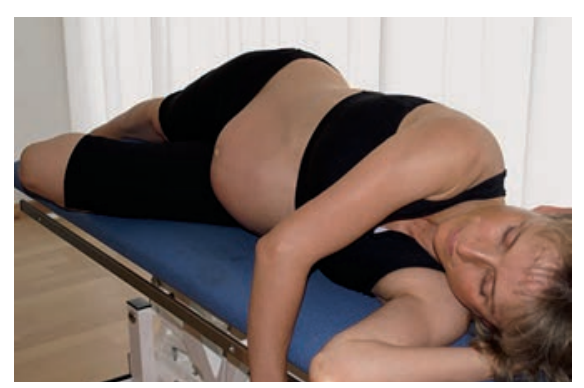

Abb. 13 Lagerung im Liegen seitlich mit angezogenem Tisch-nahem Bein.

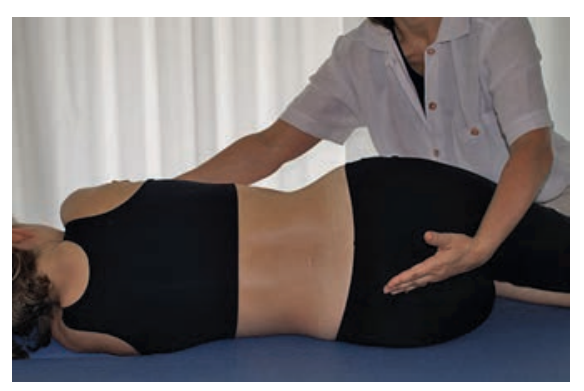

Abb. 14 Korrekte Platzierung der Hände in einer Achsel und auf dem Tisch-nahen, kaudalen SIG.

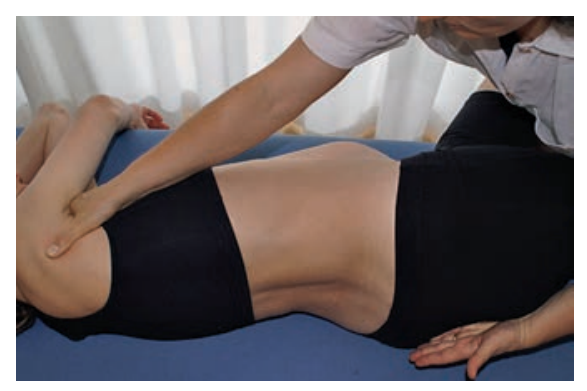

Abb. 15 Rotatorische Dehnung: dorsokraniale Streckung in der Achsel mit synchronem, ventro-kaudalen Zug auf das Tisch-nahe SIG.
Asymmetrische Spinae im Vorlaufphänomen-Test und asymmetrische Innenknöchel im BeinlängendifferenzTest zeigen die Beckenringdysfunktion an.

\section{Therapie der Beckenring- dysfunktion}

Die nachhaltige Therapie einer Beckenringdysfunktion gründet auf

- der Gelenkadjustierung,

- der ligamentären Stabilisierung und

- dem Muskelaufbau.

\section{Beckenring-Adjustierung}

Zur Gelenkadjustierung oder Deblockierung sind etliche Therapien wirksam. Dazu gehören Massage, Akupunktur, Osteopathie, Chiropraktik und Manuelle Medizin [20,21]. Physiotherapeutisch gibt es verschiedene Kombinationen aus manuellen Techniken, Mobilisation, Stabilisierung und gezieltem Muskelaufbau [22, 23]. Zur Gelenkmobilisation sind Techniken mit rotatorischer Dehnung wirksam [24]. Hierzu zählt die arthrokinematische Beckenringadjustierung, die von Dr. Bruno Maggi und mir entwickelt wurde. Sie umfasst eine Dehntechnik im Liegen und eine Impulstechnik im Stehen. Beide Techniken funktionieren gleich gut. Steht eine Gebärende (Schwangere, Wöchnerin), kann im Stehen diagnostiziert und behandelt werden, liegt sie, kann Diagnose und Therapie im Liegen erfolgen.

Bei einer Beckenverwringung (Dislokation eines Os ileum) benutze ich oft beide Techniken.

Im Folgenden wird die arthrokinematische Dehntechnik im Liegen vorgestellt (Abb. 13-15). Sie wird auf einer Bettkante, Liege oder einem Tisch durchgeführt.

Die Betroffene wird seitlich gelagert mit angezogenem Tisch-nahen Bein (Abb. 13). Der untere Arm wird unter den Kopf gelegt, der obere Arm über die Liege- oder
Bettkante fallen gelassen. In einer Wehenpause kann diese kurze Behandlung problemlos durchgeführt werden. Die Behandelnde steht zur Betroffenen gerichtet, kaudal des angezogenen Knies. Eine Hand wird in die Achselhöhle der Betroffenen gelegt und umfasst die Thoraxwand auf Höhe der Skapula (Abb. 14). Sie zieht durch einen dorso-kranialen, atemsynchronen Schub die Wirbelsäule mitsamt dem Sakrum aus dem Beckenring und dreht sie leicht nach dorsal. Gleichzeitig zieht die Hand auf dem unteren, Tisch-nahen SIG das Sakrum nach ventro-kaudal (Abb. 15). Es resultiert eine leichte Öffnung des Tisch-nahen SIG, sodass es sich selber in die physiologische Position adjustieren kann.

Dieses gleichzeitige, atemsynchrone Dehnen wird bei Nicht-Gebärenden 3-mal pro Seite durchgeführt, während beim Gebären oft eine einseitige Behandlung genügt. Nach der Behandlung wird der Beckenring kontrolliert. Bei chronischen Problemen wird die Behandlung meist nochmals wiederholt, bis eine physiologische Beckenringfunktion erreicht ist.

\section{Stabilisierung und Prophylaxe}

Unabhängig von der Dauer der bisherigen Probleme instruiere ich alle Betroffenen in drei prophylaktischen Maßnahmen: Bis zur nächsten Kontrolle (Ausnahme Geburt)

- sollen federnde Schuhe resp.

Schuheinlagen getragen werden,

- soll komplett auf Drehübungen in Freizeit und Sport (Yoga, Fitness, Tanz, Crawl, Jogging etc.) verzichtet werden und

- soll kein Abdrehen von Rumpf zum Becken im Alltag durchgeführt werden (kein Staubsaugen, kein Abdrehen auf dem Bürostuhl, kein Nach-Hinten-Schauen beim Autofahren etc.).

Manchmal genügen diese Maßnahmen zur Eigenstabilisierung des Beckenrings, oft jedoch nicht. Dann wird ein nicht- 
elastisches Beckentuch über Symphyse und die Trochanter angelegt (wie ein tiefer Beckengurt) und zur Selbstanwendung angeleitet [23]. Das Beckentuch sollte tagsüber getragen werden, bei vollständig instabilen Verhältnissen in

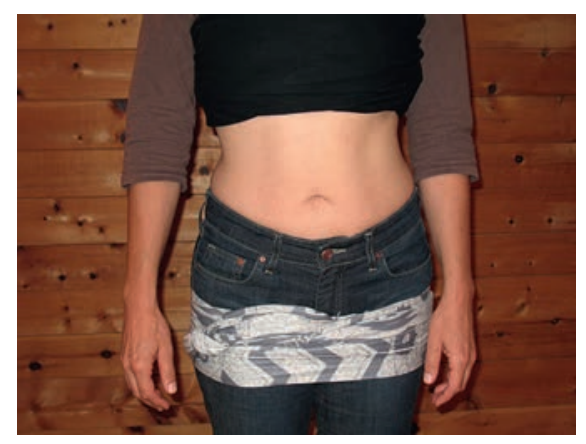

Abb. 16 Beckentuch über den Trochantern.

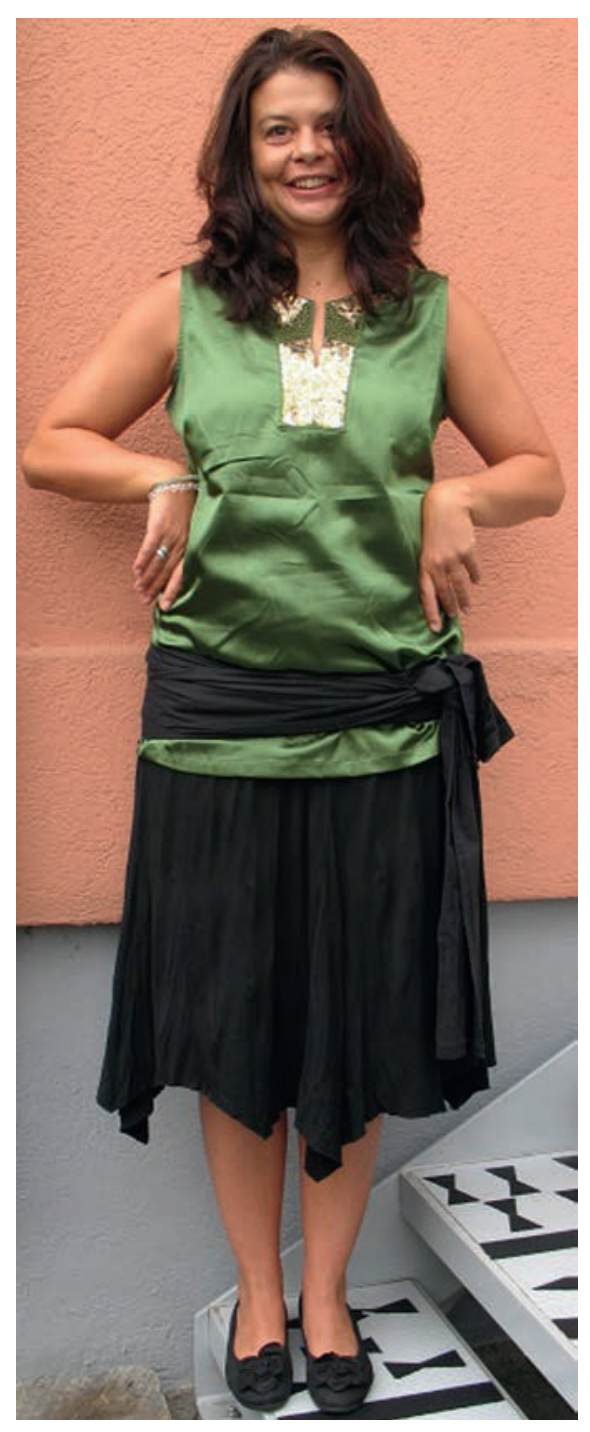

Abb. 17 Beckentuch im Alltag.

\section{Fallbeispiel}

Frau Z. hat eine schöne erste Schwangerschaft erlebt. Die Geburt beginnt zögerlich, langsam kommen gute Wehen. Frau Z $\mathrm{Z}$. ist nach einer durchwachten Nacht müde und muss viel liegen. Der Muttermund öffnet sich bis etwa acht Zentimeter, dann geht es nicht weiter. Die Wehen verändern sich, es tut mehr und mehr weh, der Kopf des Kindes drückt auf das Kreuz, und Frau Z. ist am Ende ihrer Kräfte. Sie wird weinerlich und unruhig. Weder Wärme noch Kälte helfen gegen die Kreuzschmerzen, sie verträgt keinen Handdruck vom Partner oder von der Hebamme. Das Kind bleibt mit dem Kopf auf der Interspinalebene stehen.

Frau Z. versucht, tapfer mitzumachen und steht, kauert, geht und dreht sich nach Anleitung. Pflanzliche und homöopathische Mittel helfen nur kurz. Die Schmerzen werden eher noch stärker.

Nach insgesamt 18 Std. lässt sich Frau Z. vom Geburtshaus in ein Zentrumspital verlegen. Der Kopf ist inzwischen etwas tiefer als interspinal, sodass von einer einfachen Vakuum-Entbindung ausgegangen wird. Zweimal gleitet das Vakuum ab; das Köpfchen tritt nicht tiefer. Der Versuch einer Forcepsentbindung misslingt, sodass eine Sectio caesarea durchgeführt wird. Die MRT-Aufnahme des Beckens im Wochenbett zeigt keine verengten Beckenverhältnisse. Acht Wochen nach der Geburt sehe ich Frau Z. das erste Mal. Sie hat keine Beschwerden mehr, kann sich frei bewegen und gut stillen. Allein aufgrund der Geburtsgeschichte untersuche ich den Beckenring und finde eine deutliche Verschiebung der SIG vor. Ich behandle den Beckenring arthrokinematisch und kontrolliere nach einer Woche. Der Beckenring bleibt stabil und beschwerdefrei. Ich empfehle ihr, sich frühzeitig in der nächsten Schwangerschaft bei mir zu melden.

In der zweiten Schwangerschaft treten schon früh wiederkehrende SIG-Blockaden auf, die jeweils arthrokinematisch behandelt werden. Der Partner traut sich die Technik nicht zu, auch wegen den Komplikationen unter der 1. Geburt. Da eine spontane Beckenring-Stabilisierung nach der 32. Woche eintritt, erwägt Frau Z. den erneuten Versuch einer natürlichen Geburt. Nach eingehender Beratung und Diskussion entscheidet sie sich für eine Hausgeburt. Frau Z. eröffnet in wenigen Std. fast schmerzfrei und gebärt innerhalb von 20 Min. in der Badewanne zu Hause - ein wohltuendes und heilsames Erlebnis sowohl für Mutter und Kind als auch für die ganze Familie. Der Beckenring bleibt stabil, auch im Wochenbett.

Frau Z. berichtet von einem konstanten starken Schmerz tief im rechten Unterbauch, etwa auf Höhe des rechten Leistenkanals während der ersten Geburt, der erst durch den Kaiserschnitt verschwunden ist. Sie habe angenommen, dass dieser Schmerz zu einer Geburt gehöre, aber er trat während der zweiten Geburt nie auf.

der ersten Woche auch nachts. Die teilelastischen Beckengurte, oft mit Klettverschlüssen versehen, genügen für eine ligamentäre Stabilisierung nicht $[25,26]$. Sie können aber, wie auch das Taping, die Beschwerden verringern. Allerdings wirken lediglich nicht-elastische Beckengurte und Beckentücher nachhaltig und heilen effektiv (Abb. 16, 17).
Kontrollen sollten zu Beginn wöchentlich stattfinden, nach einigen Wochen dann alle 2-4 Wochen. Insgesamt müssen die Beckenringgelenke zwölf Wochen lang durchgehend physiologisch platziert sein, um eine komplette ligamentäre Stabilisierung zu erreichen, ab der ersten Kontrolle ohne Beckenringdysfunktion gerechnet. 


\section{Muskelaufbau}

Der Muskelaufbau erfolgt in einem Stufenprogramm, wie es in der Sport-Physiotherapie üblich ist. Wird die Beckenring-stabilisierende Muskulatur zu früh trainiert, also vor einer ligamentären Stabilisierung, werden die lockeren Gelenke wieder in die alte dysfunktionelle Stellung verzogen.

Wie bei allen anderen Gelenksdislokationen sollte die muskuläre Stabilisierung erst nach der ligamentären Stabilisierung trainiert werden, die beim Beckenring erfahrungsgemäß 12 Wochen benötigt. Das Aufbauen der symmetrischen Rumpf- und Beckenmuskulatur kann jedoch schon nach 4 Wochen stabilem Beckenring beginnen [27].

Hier empfehlen wir insbesondere das Nordic Walking, das durch das gehaltene, leichte Vornüberbeugen einen Muskelaufbau im Kreuz bewirkt. Ebenso sind Liegestütze, symmetrische Rumpfbeugen und symmetrische Dehnübungen von großem Vorteil. Nach acht Wochen kommen symmetrische Bauchmuskeltrainingseinheiten dazu. Ab zwölf Wochen werden die gesamten muskulären Beckenring-Stabilisatoren in einem Stufenprogramm aufgebaut.

Bei Wöchnerinnen gehen wir erst vier Monate nach Geburt von einem stabilen Beckenring aus; erst ab dann beginnt das muskuläre Stufenprogramm. Mit Geduld und Achtsamkeit können so auch langwierige, chronische Beckenringdysfunktionen nachhaltig behoben werden.

\section{Fazit}

In den 20 Jahren als Geburtshelferin habe ich diese Problematik so oft erleben, dass ich empfehle, das Erkennen und Behandeln von Beckenringdysfunktionen in die Ausbildung von Hebammen und Geburtshelfer aufzunehmen. Diagnostik und Therapie müssen in einem praktischen Kurs erlernt und geübt werden. Bislang habe ich ca. 1200 Personen behandelt und alle erlebten eine Linderung ihrer Beschwerden und fast alle eine komplette Beckenringstabilisierung mit Beschwerdefreiheit.

Da die Beckenringdiagnostik wenig zeitaufwändig ist und keine Apparaturen benötigt, empfehle ich bei allen Schwangeren einmal pro Trimenon eine Diagnostik durchzuführen. Mit Blick aufs Gebären ist die Untersuchung kurz vor der Geburt am wichtigsten. Im Wochenbett empfehle ich vor Ablauf von vier Monaten eine Diagnostik durchzuführen. Damit kann die Rate an chronischen Beckenringdysfunktionen reduziert werden. 
Dr. med. Bruno Maggi und ich unterrichten diese Technik seit vielen Jahren. Der Schweizerische Hebammenverband und geburtshilfliche Abteilungen bieten entsprechende Kurse an (siehe www.arthrokinematik.ch).

Abb. 8-15 Fotos von Beat Schilt. Abb. 2, 16, 17 von Dorin Ritzmann

\section{Literatur}

1 Albert HB et al. Incidence of four syndromes of pregnancy-related pelvic joint pain. Spine 2002; 27 (24): 28312834

2 Kumle M et al. Use of hormonal contraceptives and occurrence of pregnancyrelated pelvic pain: a prospective cohort study in Norway. BMC Pregnancy Childbirth 2004; 4 (1): 11

3 MacLennan $\mathrm{AH}$. The role of the hormone relaxin in human reproduction and pelvic girdle relaxation. Scand J Rheumatol Suppl 1991; 88: 7-15

4 Kristiansson P et al. Back pain in in-vitro fertilized and spontaneous pregnancies. Human Reproduction 1998; vol 13 (11): 3233-3238.

5 Wang SM et al. Low back pain during pregnancy: prevalence, risk factors and outcomes. Obstet Gynecol. 2004 Jul; 104 (1): 65-70

6 Albert HB et al. Risk factors in developing pregnancy-related pelvic girdle pain. Acta Obstet Gynecol Scand. 2006; 85 (5): 539-544

7 Maggi B. Manuelle Therapie und Geburtshilfe. 1998; 11: 176-181

8 Legrand FD et al. Efficacy of exercise as an adjunct treatment for clinically depressed inpatients during the initial stages of antidepressant pharmacotherapy: An open randomized controlled trial. J Affect Disord. 2015 Nov 30; 191: 139-144

9 Stubbs B et al. Challenges Establishing the Efficacy of Exercise as an Antidepressant Treatment: A Systematic Review and Meta-Analysis of Control Group Responses in Exercise Randomised Controlled Trials. Sports Med 2015 Dec 26 (Epub ahead of print)

10 Lee D. An Integrated Model of ,joint“function and its clinical application. 4th Interdisciplinary World Congress on
Low Back \& Pelvic Pain. 2001 Nov, Montreal, 137-152

11 Abitbol MM. The shapes of the female pelvis. Contributing factors. J Reprod Med. 1996 Apr; 41 (4): 242-250

12 O'Sullivan PB et al. Changes in pelvic floor and diaphragm kinematics and respiratory patterns in subjects with sacroiliac joint pain following a motor learning intervention: a case series. Man Ther. 2007 Aug; 12 (3): 209-218

13 Nielsen LL. Clinical findings, pain descriptions and physical complaints reported by women with post-natal pregnancy-related pelvic girdle pain. Acta Obstet Gynecol Scand. 2010 Sep; 89 (9): 1187-1191

14 Buyruk HM et al. Measurement of sacroiliac joint stiffness in peripartum pelvic pain patients with Doppler imaging of vibrations (DIV). Eur J Obstet Gynecol Reprod Biol. 1999 Apr; 83 (2): 159-163

15 Damen L et al. Pelvic pain during pregnancy is associated with asymmetric laxity of the sacroiliac joints. Acta Obstet Gynecol Scand. 2001 Nov; 80 (11): 1019-1024

16 Borell $U$ et al. The movements at the sacroiliacal joints and their importance in the pelvic dimensions during parturition. Acta Obst et Gynaecol Scand 1957, 36: 42-57

17 Noren L et al. Lumbar back and posterior pelvic pain during pregnancy: a 3-year follow-up. Eur Spine Journal Feb 2004; 267-271

18 Nilsson-Wikmar L. Back pain postpartum: clinical and experimental studies. Dissertation 2003.

19 Commissaris DA et al. Joint coordination during whole-body lifting in women with low back pain after pregnancy. Arch Phys Med Rehabil. 2002 Sep; 83 (9): 1279-1289

20 Elden $\mathrm{H}$ et al. Effects of acupuncture and stabilising exercises as adjunctive treatment in pregnant women with pelvic girdle pain: a randomised single blind controlled trial. BMJ 2005 April 2: 330 (7494): 761

21 Elden $\mathrm{H}$ et al. Regression of pelvic girdle pain after delivery: follow-up of a randomised single blind controlled trial with different treatment modalities. Acta Obstet Gynecol Scand. 2008; 87 (2): 201-208
22 Stuge B et al. The efficacy of a treatment program focusing on specific stabilizing exercises for pelvic girdle pain after pregnancy. Spine 2004. Vol 29 (4): 351-359

23 Lee D. An Integrated Model of ,joint“function and its clinical application. 4th Interdisciplinary World Congress on Low Back \& Pelvic Pain. 2001 Nov, Montreal, 137-152

24 Hakata S et al. Wirksamkeit der AK-Hakata-Methode bei der Behandlung der akuten Lumbago. Manuelle Medizin 2005 Febr (1): 19-22

25 Mens JM et al. The mechanical effect of a pelvic belt in patients with pregnancyrelated pelvic pain. Clin Biomech (Bristol, Avon). 2006 Feb; 21 (2): 122-127

26 Vleeming A et al. An integrated therapy for peripartum pelvic instability: a study of the biomechanical effects of pelvic belts. Am J Obstet Gynecol. 1992 Apr; 166 (4): 1243-1247

27 Stuge B et al. The efficacy of a treatment program focusing on specific stabilizing exercises for pelvic girdle pain after pregnancy. Spine 2004. Vol 29 (4): 351-359

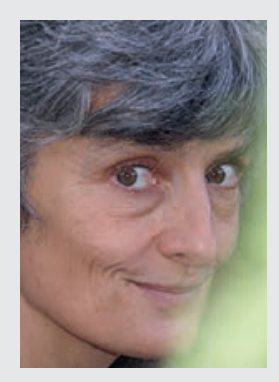

Dorin Ritzmann

Fachärztin Gynäkologie und

Geburtshilfe

Medizin Feminin AG

Austraße 35a

8953 Dietikon/CH

E-Mail: dorin.ritzmann@

medizinfeminin.ch 